\title{
No evidence for surface organization in Kanizsa configurations during continuous flash suppression
}

\author{
Pieter Moors ${ }^{1} \cdot$ Johan Wagemans $^{1} \cdot$ Raymond van Ee $^{1,2,3} \cdot$ Lee de-Wit $^{1}$
}

Published online: 24 December 2015

(C) The Psychonomic Society, Inc. 2015

\begin{abstract}
Does one need to be aware of a visual stimulus for it to be perceptually organized into a coherent whole? The answer to this question regarding the interplay between Gestalts and visual awareness remains unclear. Using interocular suppression as the paradigm for rendering stimuli invisible, conflicting evidence has been obtained as to whether the traditional Kanizsa surface is constructed during interocular suppression. While Sobel and Blake (2003) and Harris, Schwarzkopf, Song, Bahrami, and Rees (2011) failed to find evidence for this, Wang, Weng, and He (2012) showed that standard configurations of Kanizsa pacmen would break interocular suppression faster than their rotated counterparts. In the current study, we replicated the findings by Wang et al. (2012) but show that neither an account based on the construction of a surface nor one based on the long-range collinearities in the standard Kanizsa configuration stimulus could fully explain the difference in breakthrough times. We discuss these findings in the context of differences in the amplitudes of the Fourier orientation spectra for all stimulus types. Thus, we find no evidence that the integration of separate elements takes place during interocular suppression of Kanizsa stimuli, suggesting that this Gestalt involving figure-ground assignment is
\end{abstract}

Pieter Moors

pieter.moors@ppw.kuleuven.be

1 Laboratory of Experimental Psychology, Department of Brain \& Cognition, University of Leuven, Tiensestraat 102, box 3711, B-3000 Leuven, Belgium

2 Donders Institute, Department of Biophysics, Radboud University, Nijmegen, The Netherlands

3 Department of Brain, Body \& Behavior, Philips Research Laboratories, Eindhoven, The Netherlands not constructed when rendered nonconscious using interocular suppression.

Keywords Visual awareness - Continuous flash suppression . Figure-ground organization $\cdot$ Illusory contours $\cdot$ Kanizsa stimulus

\section{Introduction}

Our phenomenological experience consists of a perceptually organized whole in which the signals sent from the retina have been structured into a meaningful scene composed of distinct objects and surfaces (Palmer, 1999). This process of perceptual organization occurs in accordance with a set of grouping principles, including proximity, similarity, good continuation, common fate, connectedness, or closure (Wagemans et al., 2012). Given the apparent ease with which perceptual grouping happens, several studies have tested the extent to which it operates independently from attention and/or awareness (Alais \& Blake, 2015; Gillebert \& Humphreys, 2015; Schwarzkopf \& Rees, 2015). Perhaps unsurprisingly, the results of these studies paint a complicated picture, with the results often being contingent on the exact paradigm used to render stimuli nonconscious and with many different perceptual organizational processes being involved. It is probably fair to conclude, however, that there is evidence that some forms of perceptual organization still take place in the absence of attention (Gillebert \& Humphreys, 2015; Kimchi, 2009; Moore \& Egeth, 1997). Likewise, there also is some evidence that certain forms of perceptual organization still manifest themselves in the absence of awareness (Mitroff \& Scholl, 2005; Montoro, Luna, \& Ortells, 2014; Norman, Heywood, \& Kentridge, 2013). 
The question, therefore, becomes what kinds of perceptual organization can occur without attention or awareness, and to what extent does this depend on the paradigm used to render stimuli invisible. In this study, we focus on the Kanizsa stimulus, using a paradigm for suppressing stimuli from awareness that should disrupt higher level processing.

Previous work from our lab has highlighted a potential distinction between perceptual organizational phenomena that involve the grouping of elements in a display and the assignment of figure-ground relationships (Machilsen \& Wagemans, 2011; Vancleef et al., 2015; Vancleef, Wagemans, \& Humphreys, 2013). Both contour grouping and figureground assignment have been shown to contribute to the perception of a surface when presented with the standard Kanizsa stimulus (Conci et al., 2009; Kogo, Strecha, Van Gool, \& Wagemans, 2010; Kogo \& Wagemans, 2013), yet the contribution of both has been shown to be different with respect to timing and neural correlates (Cox \& Maier, 2015; Poort et al., 2012). Indeed, there is evidence that the perceptual organization involved in the construction of a Kanizsa surface is dependent upon the functioning of higher areas in the visual ventral stream, namely the lateral occipital complex (LOC) (de-Wit, Kentridge, \& Milner, 2009; Seghier \& Vuilleumier, 2006; Stanley \& Rubin, 2003). Other studies have documented activity in areas, such as V1 and V2 when perceiving Kanizsa figures, but the latency of these neural responses suggests that feedback processes are involved (Lee \& Nguyen, 2001; von der Heydt, Peterhans, \& Baumgartner, 1984). Indeed, a recent TMS study by Wokke, Vandenbroucke, Scholte, and Lamme (2013) suggests that feedback to early visual areas is critical for surface perception in the Kanizsa stimulus. In sum, there is evidence for a distinction between contour grouping and the perception of figure-ground relationships, and that figure-ground perception for the Kanizsa figure in particular relies on processing at higher stages of the ventral stream.

Parallel to this, a paradigm for rendering stimuli invisible, called "continuous flash suppression (CFS)," is known to have a large impact in reducing the flow of visual input to higher visual areas, such as LOC (Fang \& He, 2005; Hesselmann \& Malach, 2011). This paradigm renders stimuli invisible by continuously flashing stimuli to one eye, rendering the input to the other eye inaccessible to awareness for extended periods of time (Tsuchiya \& Koch, 2005). Given this theoretical background, it would seem logical to predict that it would be impossible for this Gestalt to be constructed or influence behavior when suppressed from awareness using CFS (see also Alais and Blake, 2015), because the flow of activity to higher areas involved in the construction of the Kanizsa stimulus is blocked with CFS. The evidence however, is currently mixed.

In the first study on the perception of Kanizsa figures using CFS, Harris, Schwarzkopf, Song, Bahrami, and Rees (2011) showed that observers were unable to discriminate in which direction a suppressed Kanizsa triangle configuration was pointing, yet could do so perfectly when the stimulus was visible. This is consistent with previous work using binocular rivalry, in which Sobel and Blake (2003) set out to test whether the perception of so-called illusory contours (induced by certain Kanizsa stimuli) had similar effects on initiating switches between eyes compared to contours derived from physically presented contrast edges. When physically presented edges moved over a suppressed stimulus, the suppressed stimulus became visible to the participant, whereas this did not happen for configurations that elicited a contour percept that did not derive from a physically presented edge. In a more recent study, however, Wang, Weng, and He (2012) used a variant of the CFS (the breaking-CFS or b-CFS) paradigm to measure how long it took for configurations of standard pacmen (that are able to induce a surface percept when fully visible) to overcome suppression induced by CFS. In apparent contrast with the previous studies, Wang et al. (2012) observed that configurations able to induce a triangular Kanizsa surface break suppression faster than non-surfaceinducing control conditions (even when controlling for mirror symmetry in the control stimulus). Wang et al. (2012) suggested that this result provided evidence that the processes involved in constructing the Kanizsa surface are able to manifest even when suppressed from awareness using CFS. ${ }^{1}$ This result would further suggest that some forms of figure-ground assignment might be possible in the absence of perceptual awareness, even while little visual input is able to reach higher areas of the ventral stream thought to mediate these processes.

Given the potential importance of this result, we first set out to replicate the result by Wang et al. (2012) using the b-CFS paradigm. We then tested two different sets of control stimuli to disentangle the contribution of the alignment of the edges in the image and the potential to perceive a surface induced by different variants of the Kanizsa configuration. Indeed, in the original Wang et al. study, no attempt was made to dissociate contributions of edge alignment from the perception of a surface. That is, for the configurations in which a surface could be perceived, the edges of the individual elements were always co-linear to each other (which is known to make a distinct contribution, see Conci et al., 2009). To control for the role of edge alignment in the image, we used a cross stimulus (instead of the standard pacmen) in which there is the same degree (or perhaps more) alignment between the edges in the image, but in which a Kanizsa surface is not normally elicited even when fully visible (Fig. 1A, third column). If the suppression time difference observed in Wang

\footnotetext{
${ }^{1}$ Note that Wang et al. (2012) interpreted their results explicitly in terms of unconscious processing, the validity of which has been debated by Stein and Sterzer (2014) and by Gayet, van der Stigchel, and Paffen (2014).
} 


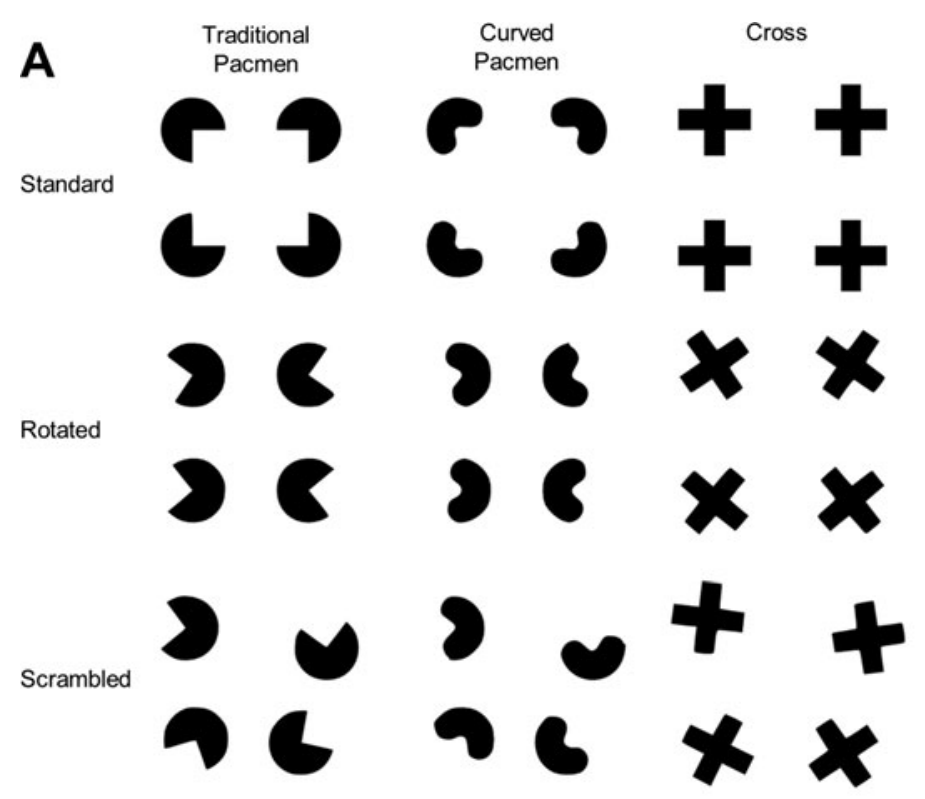

B

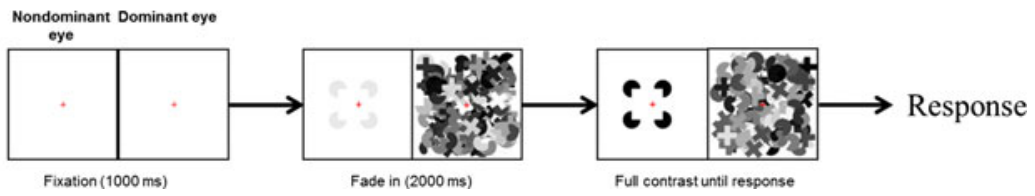

Fig. 1 (A) Stimulus set used in Experiments 1 (two top rows) and 2 (all rows). (B) Basic trial sequence used in all experiments. A fixation cross was presented for $1,000 \mathrm{~ms}$ after which the CFS mask was displayed in the dominant eye and the suppressed stimulus in the nondominant eye.
The suppressed stimulus was gradually increased in contrast over the course of 2,000 ms after which it remained at full contrast until it broke suppression et al. (2012) was due to the alignment of the edges rather than the construction of a surface (and engagement of figureground assignment), then the suppression time difference for a standard (aligned) and rotated (non-aligned) cross stimulus should be predicted to be similar to the one seen for the traditional Kanizsa stimulus. A second control stimulus was developed based on the stimuli used by Stanley and Rubin (2003), in which a surface percept is evoked by curved pacmen. The edges in these curved pacmen are obviously not collinear like those in the traditional Kanizsa or the cross stimuli, yet the stimulus still evokes a surface percept when fully visible (Fig. 1A, middle column). If the potential for the stimulus to elicit a surface percept contributes to the suppression time difference observed for the traditional pacmen configuration, then the suppression time difference for the curved pacmen should be of a similar magnitude.

\section{Experiment 1}

In Experiment 1, we manipulated Stimulus Type (traditional pacmen, cross, or curved pacmen) and Configuration Type (standard or rotated). The comparison of a standard and rotated traditional pacmen configuration enables us to replicate the results from Wang et al. (2012), whilst the inclusion of a cross stimulus and curved pacmen (both also in standard and rotated configurations) enables us to test the relative contribution of edge collinearity and surface construction to any potential differences in suppression time. Furthermore, given that the surface percept in the curved pacmen configuration is weaker than the one elicited by the traditional pacmen configuration (Stanley \& Rubin, 2003), one could predict that, if a surface is constructed during interocular suppression, breakthrough times would vary according to surface strength. Our predictions can be summarized as follows:

- If the configuration effect (standard vs. rotated) is due to the ability to induce a Kanizsa surface during CFS, we predict an effect of configuration that does not differ between the traditional versus curved pacmen but does differ between traditional pacmen and cross stimuli, as well as between curved pacmen and cross stimuli.

- If the configuration effect (standard vs. rotated) is due to the existence of aligned edges, we predict an effect of configuration that does not differ between the traditional 
versus cross stimuli but does differ between traditional and curved pacmen, as well as between cross stimuli and curved pacmen.

In all conditions, observers were presented with a stimulus in one eye rendered invisible by the presentation of a flashing CFS stimulus (at $10 \mathrm{~Hz}$ ) presented to the other eye. The critical dependent variable in all conditions was the time taken to "break" this suppression, such that participants could reliably report the location of the previously suppressed stimulus.

\section{Methods}

\section{Participants}

Twenty people (age range: $18-30$ years) participated in the experiment in exchange for monetary compensation. All participants had normal or corrected-to-normal vision and were naïve with respect to the goal of the study. The study was approved by the local ethical committee and all participants provided informed consent at the start of the experiment.

\section{Apparatus}

Stimuli were shown on two 19.8-in Sony Trinitron GDM F500-R (2048 × 1536 pixels at $60 \mathrm{~Hz}$, for each) monitors driven by a DELL Precision T3400 computer with an Intel Core Quad CPU Q9300 2.5-GHz processor running on Windows XP. Binocular presentation was achieved by a custom made stereo set-up. Two CRT monitors, which stood opposite to each other (distance of $220 \mathrm{~cm}$ ), projected to the left and right eye respectively via two mirrors placed at a distance of $110 \mathrm{~cm}$ from the screen. A head-and-chin rest (15 cm from the mirrors) was used to stabilize fixation. The effective viewing distance was $125 \mathrm{~cm}$. Stimulus presentation, timing, and keyboard responses were controlled with custom software programmed in Python using the PsychoPy library (Peirce, 2007, 2009).

\section{Stimuli}

The background of the display consisted of a random checkerboard pattern to achieve stable binocular fusion. The individual elements of the checkerboard were $0.34^{\circ}$ by $0.34^{\circ}$. In both eyes, a white frame $\left(10^{\circ}\right.$ by $\left.10^{\circ}\right)$ where the stimuli would be presented was superimposed on the checkerboard pattern. A black (eye dominance measurement) or red (main experiment) fixation cross was continuously present during the experiment (size $0.5^{\circ}$ by $0.5^{\circ}$ ). In the eye dominance measurement phase, the target consisted of an arrow (maximal width $4^{\circ}$, maximal height $2^{\circ}$ ) and the CFS mask consisted of 150 squares with randomly picked sizes between $1^{\circ}$ and $2^{\circ}$ and a random luminance value.

The radius of the traditional pacman stimulus was $0.8^{\circ}$ and the diagonal distance between the pacmen was $2^{\circ}$. The cross stimulus consisted of four crosses and was created such that the overall density of each cross matched that of the traditional pacmen stimuli $\left(1.75^{\circ} 00 \mathrm{D} 71.75^{\circ}\right)$. The curved pacmen stimulus was created by superimposing two small discs (radius $0.34^{\circ}$ ) on the half of a full disc on which a white ellipse was drawn (major radius $1.19^{\circ}$, minor radius $0.37^{\circ}$ ). For all three stimuli, the area of the inner surface between the inducers was as equal as possible (exactly the same for the traditional pacmen and cross stimulus and slightly different for the curved pacmen stimulus). The rotated versions of the stimulus were generated by rotating the individual elements $45^{\circ}$ with a jitter of $\pm 10^{\circ}$ (Fig. 1A, middle row).

Given the importance in feature overlap between mask and stimuli in determining the effectiveness of CFS (Hong \& Blake, 2009; Maehara, Huang, \& Hess, 2009; Moors, Wagemans, \& de-Wit, 2014; Yang \& Blake, 2012) and the fact that the stimuli in the different conditions differed in various ways, we did not use a traditional Mondrian style CFS mask in the main experiment. Indeed, pilot results using a classical CFS mask indicated prolonged suppression for the cross condition, containing only vertical and horizontal orientations as in the traditional Mondrian CFS mask, indicating feature-selective depth of suppression (Yang \& Blake, 2012). Therefore, we generated CFS masks that consisted of the individual elements of all different stimuli (Fig. 1B). This set of masks was generated before the start of the experiment (but was kept the same for every participant) and on each refresh (i.e., every 100 milliseconds) a new mask was selected from the pool with the restriction that the previous mask could not be used twice in a row.

\section{Procedure}

Before starting the main experiment, participants' eye dominance was measured using the method set out by Yang, Blake, and McDonald (2010). On every trial, a fixation cross was presented for $1000 \mathrm{~ms}$. Next, an arrow was presented in one eye and the CFS mask in the other (10-Hz refresh rate). Participants had to indicate the direction of an arrow as soon as it broke through the CFS mask by pressing "1" (for left) or "3" (for right) on a numerical keyboard. The CFS mask was randomly presented to the left or right eye (40 trials per eye) and after 80 trials eye dominance was determined as the eye in which the mean suppression time of the arrow was lowest. In all subsequent parts of the experiment, the CFS mask was presented to the participants' dominant eye.

During the main experiment, participants were presented with the different stimuli from Fig. 1A (first two rows only) of which the position relative to fixation was manipulated on 
each trial (i.e., shifted $0.5^{\circ}$ left-, right-, up-, or downwards). During the rest of the experiment, the participants always had to indicate as fast as possible in which direction the target stimulus was shifted by pressing " 2, ," "4," "6," or "8" on a numerical keyboard for down, left, right, and up, respectively. Every trial started with a 1-second fixation period after which the target stimulus was presented to the nondominant eye and the CFS mask to the dominant eye (refreshing at $10 \mathrm{~Hz}$ ). The contrast of the target stimulus increased linearly from 0 to $100 \%$ over $2000 \mathrm{~ms}$ and then remained at full contrast until the stimulus was detected. Participants first completed a practice block in which the stimuli were presented without suppression in order to acquaint them with the task. In a second practice block, the CFS mask was presented together with the target stimuli. If everything was clear after the two practice blocks, participants began the actual experiment.

\section{Design}

The procedure to assess eye dominance included 80 trials. The two practice blocks contained 24 trials each. The main experiment consisted of 192 trials in total. The design of the main experiment was a full factorial $3 \times 2$ within-subjects design with three levels of the Stimulus Type factor (traditional pacmen, cross, and curved pacmen) and two levels of the Configuration Type factor (two levels: standard, rotated). The order of all experimental conditions was always randomized. After each sequence of 64 trials, participants were instructed to take a break of at least 1 minute.

\section{Results}

Correct suppression times (95\% of the data) were analyzed after log-transforming them due to the positive skew in the suppression time distributions. Outliers were defined as three times the standard deviation of the mean suppression time and these were removed from the data set ( $2 \%$ of the data). Most of the outlying data points were presumably due to extremely effective suppression. The mean suppression times for each condition are summarized in Fig. 2.

Statistical inference was done in a Bayesian framework relying on model selection using Bayes Factors (Rouder \& Morey, 2012; Rouder, Morey, Speckman, \& Province, 2012; Rouder, Speckman, Sun, Morey, \& Iverson, 2009). The Bayes Factor quantifies the relative degree of evidence of one statistical model over another and therefore provides an intuitive measure to quantify the degree of belief in one statistical model over another (e.g., a model with and without a main effect of Configuration Type). The $\mathrm{R}$ package BayesFactor (version 0.9.9) was used to compute the Bayes Factors (Morey \& Rouder, 2015). All considered models were ANOVA style models including random intercepts for participants, and the

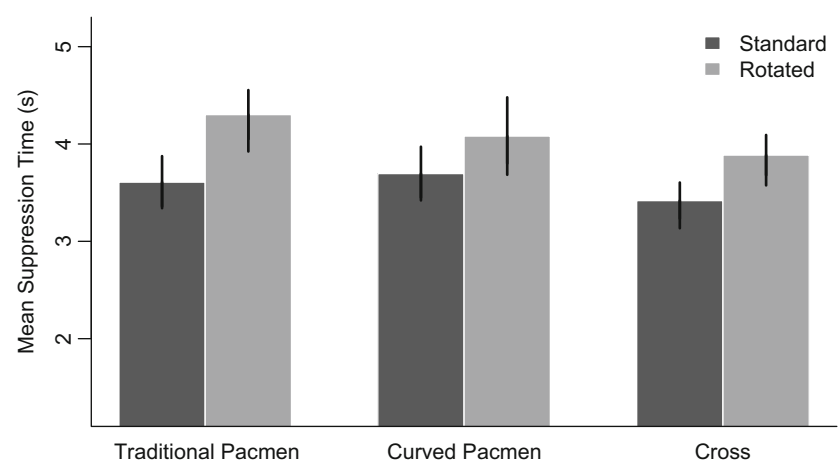

Fig. 2 Results of Experiment 1. The bars depict the mean suppression time for each condition. The error bars denote $95 \%$ within-subject confidence intervals using the procedure set out by Morey (2008).

default settings for the priors (medium prior scale for fixed effects and nuisance prior scale for the participant effect). As a guideline to interpret the resulting Bayes Factors, we use the classification proposed by Jeffreys (1961) in that Bayes Factors from 3 onwards constitute substantial evidence for one model over the other.

The Bayes Factor analysis is summarized in Table 1. The best fitting model is depicted as having a Bayes Factor of 1 and all the other Bayes Factors can be interpreted as how much more likely the best model is compared to another model. As is apparent from Table 1, the preferred model includes main effects of both Stimulus Type and Configuration Type, and no interaction between these factors. Indeed, a model including an interaction is 53 times less likely as a model not including it. The main effect of Stimulus Type was largely due to the cross stimulus breaking suppression faster than the other stimuli. The main effect of Configuration Type indicates that standard stimuli broke suppression faster, on average, than rotated stimuli.

\section{Discussion}

In Experiment 1, we replicated the suppression time difference between a standard and rotated version of the traditional pacmen stimulus, in line with the results of Wang et al. (2012). However, this configuration effect did not interact

Table 1 Bayes Factor analysis for Experiment 1

\begin{tabular}{ll}
\hline Model & Bayes Factor \\
\hline $\mathrm{ST}+\mathrm{CT}$ & 1 \\
$\mathrm{ST}+\mathrm{CT}+\mathrm{ST}^{*} \mathrm{CT}$ & 53 \\
All other models & $>100$ \\
\hline
\end{tabular}

Note. All Bayes Factors are relative to the best fitting model (i.e., in this case, the model of which the Bayes Factor is 1). All other models include all combinations from an empty to a full model not reported in the table. $\mathrm{ST}=$ Stimulus Type CT $=$ Configuration Type 
with the type of stimulus that was used. That is, it was statistically indistinguishable from the configuration effect observed in the curved pacmen and cross conditions. Therefore, the difference between the standard and rotated traditional pacmen conditions is presumably not driven by the potential for the stimulus to induce a surface percept or the alignment of the edges during interocular suppression. Indeed, irrespective of which process exactly contributes to the observed differences between conditions, the results of Experiment 1 indicate that the advantage for the standard configuration is not specific to the traditional pacmen configuration.

Given the pattern of results observed in Experiment 1, we were interested to explore further two potential explanations for the observed advantage for the standard configuration in a second experiment. That is, one could argue that the advantage for the standard Kanizsa was due to the configural relationship between the four elements. Alternatively, the observed advantage for the standard configuration could be due to independent contributions of the single elements comprising the configuration, based on their individual locations in the visual field and their orientation. For example, we know that observers are more sensitive to cardinal (horizontal and vertical) orientations compared with oblique ones (Appelle, 1972; Campbell \& Kulikowski, 1966) and that these cardinal orientations are over-represented in the visual cortex $(\mathrm{Li}$, Peterson, \& Freeman, 2003; Yacoub, Harel, \& Ugurbil, 2008). Thus, it could be that differences in the number of different orientations are directly influencing suppression strength.

Based on these two potential explanations, we included a scrambled version of each stimulus type in the stimulus set (Fig. 1A, third row) and repeated the experiment in a different set of observers. This scrambled version was created also by manipulating the orientation of the individual elements as well as the distance from the center of the configuration. To more directly test whether differences were being driven by the differences between each of the individual elements (rather than their configuration), we also conducted a second experiment (with the same observers), in which we measured suppression times for the individual elements of each of the different configurations (cross, traditional pacman, and curved pacman).

\section{EXPERIMENT 2}

The primary goal of Experiment 2 was to replicate the main finding from Experiment 1: that neither the ability to induce a Kanizsa surface, nor the collinearity of the lines increased the likelihood of breakthrough during CFS. The addition of two new conditions enabled us to ask two additional questions regarding the idea that surface completion mechanisms influence suppression times in CFS. First, a "scrambled" condition was added to the "standard" and "rotated" conditions to test whether more deviation from the "standard" configuration would further increase suppression times. Second, an "individual element" condition was included for all of the stimulus configurations (standard, rotated, and scrambled) to test whether any differences evident in the "full configuration" also might be evident in the "single element" condition. In addition to the predictions formulated for Experiment 1, our predictions for Experiment 2 can be summarized as follows:

- If the configuration effect observed in Experiment 1 reflects the configural relationship between the four elements, we predict that the scrambled configuration will yield slower breakthrough times compared to the standard and rotated configurations.

- If the configuration effect observed in Experiment 1 is due to the independent contributions of the four elements comprising the configuration, we predict that any effects of Configuration Type also will be evident in the single element conditions and that there will be no interaction between the Configuration Type and the Number of Elements (full configuration versus single elements).

\section{Methods}

\section{Participants}

Twenty-four people (age range: 18-30 years) participated in the experiment and received monetary compensation for their participation. All participants had normal or corrected-tonormal vision and were naïve with respect to the goal of the study. The study was approved by the local ethical committee, and all participants provided informed consent at the start of the experiment. Three participants had to be excluded, because suppression was too effective in either of the two sessions, with breakthrough times being three standard deviations longer than the mean value for all observers. One participant did not return for the second session and therefore was excluded from the analysis.

\section{Apparatus}

The experimental setup was the same as in Experiment 1.

\section{Stimuli}

The stimuli were the same as in Experiment 1, except that a scrambled version was added to the Configuration Type factor and a single element condition was developed. To create the scrambled condition, we picked four different angles relative 
to fixation at which the individual elements would be drawn and four different orientations of the individual elements, kept constant across different Stimulus Types. We refer to this additional manipulation as the scrambled configuration (in contrast to the standard and rotated conditions). This stimulus set also is depicted in Fig. 1 (third row). It should be noted that only one scrambled exemplar was created for this condition, being the one shown in Fig. 1A. This choice allows us to avoid a difference in familiarity between the different stimuli, because there also is only one exemplar in the other conditions.

In the single element condition, we presented individual elements (just one pacman or cross rather than all four) at one of the same locations at which they were presented in the full configuration experiment.

\section{Procedure}

The experimental procedure was exactly the same as in Experiment 1, except for the session in which the individual elements of the stimuli were presented, in which the task was changed. In this condition, participants had to indicate in which quadrant of the display the stimulus was presented by pressing " 1, ," "3," "4," or " 6 " for bottom left, bottom right, up left, and up right, respectively.

\section{Design}

The design of the experiment was a $3 \times 3 \times 2$ full-factorial within-subjects design with three levels of the Stimulus Type factor (traditional pacmen, curved pacmen, and cross), three levels of the Configuration Type factor (standard, rotated, and scrambled), and two levels of the Number of Elements factor (full configuration and single element). Participants completed 288 trials in total for the main experiment in both the full configuration as well as the single element session. The practice blocks consisted of 36 trials each. After completing the first session, participants returned a week later to complete the second session. The ordering in which the sessions were completed was randomized across participants.

\section{Results}

The data were analyzed in the same way as in Experiment 1. We removed outliers (full configuration session: 1.6\%; single element session: $1.5 \%$ ) defined as higher than three standard deviations from the mean and analyzed only correct (full configuration session: 94\%; single element session: 97\%) suppression times after logarithmically transforming them. The results for the full configuration and single element sessions are depicted in Fig. 3.
A first goal was to assess whether the results were similar to those in Experiment 1. Therefore, we first report an analysis of only the conditions used in Experiment 1 (summarized in Table 2). As the analysis indicates, the preferred model is now one including an interaction between Stimulus Type and Configuration Type on top of the main effects of Stimulus Type and Configuration Type. Follow-up analyses indicated that this interaction was mostly driven by a tendency towards the absence of a configuration effect in the curved pacmen condition $(\mathrm{BF}=2.5$ in favor of the null model). Indeed, a configuration effect was still obtained in both the traditional pacmen as well as the cross condition (both BFs > 100 in favor of a configuration effect). Thus, if only a subset of the data similar to the data observed in Experiment 1 is considered, the data obtained in Experiment 2 indicate that the configuration effect is mostly driven by the existence of aligned edges in the traditional pacmen and cross configurations. We now turn to an analysis of the full data set.

The next analysis focuses on the two predictions outlined for Experiment 2 (i.e., influence of scrambled configuration, and the difference between single and full element sessions). To assess the similarity of the results obtained in both single and full element sessions, we combined the data from both sessions and included an extra factor called Number of Elements in the analysis (i.e., full configurations vs. single elements). The results of the BF analysis are depicted in Table 3. As in Experiment 1, the best fitting model included main effects of Stimulus and Configuration Type but now also included an interaction between those factors as well as a main effect of Number of Elements (i.e., stimuli consisting of four elements broke suppression faster than those consisting of a single element). However, it should be noted that the best model in the combined analysis was only slightly preferred $(\mathrm{BF}=1.5)$ over a model including an interaction between Configuration Type and Number of Elements.

Given that the BF analysis did not distinguish between the model with and without the interaction between Configuration and Number of Elements $(\mathrm{BF}=1.5)$, we analyzed the more complex model further (i.e., the one including the interaction). Because the interpretation of this model was complicated by the interaction between Configuration and Stimulus Type, we probed the interaction between Configuration Type and Number of Elements for each Stimulus Type separately. This analysis explicitly addresses the question whether the number of elements (full configuration vs. single element) modulated the effect of Configuration for each Stimulus Type separately and is summarized in Table 4.

In the case of the traditional pacmen, the $\mathrm{BF}$ analysis does not distinguish between main effects of Configuration Type and Number of Elements or a model also including their interaction $(\mathrm{BF}=1.82)$. When this interaction is further resolved (i.e., split up by Number of Elements), strong evidence for a configuration effect is found for the full configuration session 

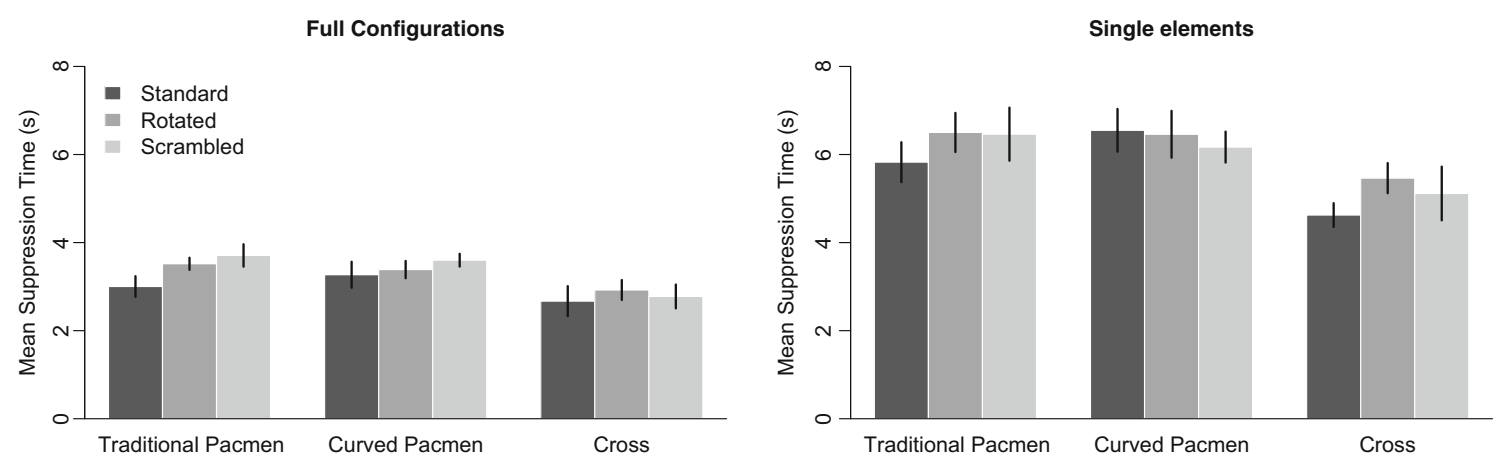

Fig. 3 Results of Experiment 2. The bars depict the mean suppression time for each condition. The error bars denote $95 \%$ within-subject confidence intervals according to Morey (2008). Full configuration session (left); single elements sessions (right)

$(\mathrm{BF}>100)$, yet the reverse is observed in the single element session. The $\mathrm{BF}$ indicates convincing evidence in favor of the null model $(\mathrm{BF}=7)$. As is apparent from Fig. 3, for the full configuration experiment, the mean suppression durations are in the direction predicted if the global configuration plays a key role (i.e., the scrambled configuration yielded the longest suppression durations). Although a similar pattern is observed in the single element experiment, the $\mathrm{BF}$ analysis indicated evidence for the absence of any differences between conditions.

In the case of the curved pacmen, the preferred model is one that includes a main effect of Number of Elements only. The pattern of results is in the direction based on a prediction of the influence of the global configuration (in that the scrambled condition is slowest to break suppression) but interestingly this pattern is completely reversed in the single element session. Analyzing both experiments together, however, does not yield sufficient evidence for an interaction between Configuration Type and Number of Elements and actually indicates no effect of configuration. Thus, for the curved pacmen, the results are in accordance with the prediction of the single-element account, in that the type of experiment did not interact with a potential configuration effect.

For the cross stimulus, the $\mathrm{BF}$ analysis indicates strong evidence for a model including a main effect of Configuration Type and Number of Elements, yet no interaction between both factors $(\mathrm{BF}=31)$. In this case, the data are

Table 2 Bayes Factor analysis for the full configuration session of Experiment 2 (only the conditions used in Experiment 1 are included)

\begin{tabular}{ll}
\hline Model & Bayes Factor \\
\hline $\mathrm{ST}+\mathrm{CT}+\mathrm{ST}^{*} \mathrm{CT}$ & 1 \\
$\mathrm{ST}+\mathrm{CT}$ & 19 \\
All other models & $>100$ \\
\hline
\end{tabular}

Note. All Bayes Factors are relative to the best fitting model (i.e., in this case, the model of which the Bayes Factor is 1). All other models include all combinations from an empty to a full model not reported in the table. $\mathrm{ST}=$ Stimulus Type CT $=$ Configuration Type not consistent with the prediction that the scrambled condition would yield the slowest suppression durations (i.e., the rotated stimuli yielded the slowest durations). However, the absence of an interaction indicates that the configuration effect did not vary according to the Number of Elements, suggesting that the differences seen in the full configuration might be driven by the same processes influencing suppression in the single element condition.

\section{Discussion}

The goal of Experiment 2 was to explore whether the configuration effect observed in Experiment 1 was due to the global characteristics of the stimulus or whether the differences could arise based on the differences in the individual elements that make up these configurations. To this end, a scrambled stimulus was included in Experiment 2 and participants were then tested in two experimental sessions, one in which the full configurations were presented and another in which the single elements of the configurations were used in isolation. We predicted that, if the global configuration was mostly responsible for the configuration effect observed in Experiment 1, the configurations of the scrambled condition would show slower suppression times compared to the standard and

Table 3 Bayes Factor analysis for both experiment types combined

\begin{tabular}{|c|c|}
\hline Model & Bayes Factor \\
\hline $\mathrm{ST}+\mathrm{CT}+\mathrm{ST} * \mathrm{CT}+\mathrm{NE}$ & 1 \\
\hline $\mathrm{ST}+\mathrm{CT}+\mathrm{ST}^{*} \mathrm{CT}+\mathrm{NE}+\mathrm{CT} * \mathrm{NE}$ & 1.5 \\
\hline $\mathrm{ST}+\mathrm{CT}+\mathrm{ST}^{*} \mathrm{CT}+\mathrm{NE}+\mathrm{ST} * \mathrm{NE}$ & 20 \\
\hline $\mathrm{ST}+\mathrm{CT}+\mathrm{ST}^{*} \mathrm{CT}+\mathrm{NE}+\mathrm{CT} * \mathrm{NE}+\mathrm{ST} * \mathrm{NE}$ & 31 \\
\hline All other models & $>100$ \\
\hline
\end{tabular}

Note. All Bayes Factors are relative to the best fitting model (i.e. in this case, the model of which the Bayes Factor is 1). All other models include all combinations from an empty to a full model not reported in the table. $\mathrm{ST}=$ Stimulus Type CT $=$ Configuration Type; $\mathrm{NE}=$ Number of Elements 
Table 4 Bayes Factor analysis of Configuration and Number of Elements, separately for each Stimulus Type

\begin{tabular}{|c|c|c|c|c|c|}
\hline \multicolumn{2}{|l|}{ Traditional pacmen } & \multicolumn{2}{|l|}{ Curved pacmen } & \multicolumn{2}{|l|}{ Cross } \\
\hline Model & Bayes Factor & Model & Bayes Factor & Model & Bayes Factor \\
\hline $\mathrm{CT}+\mathrm{NE}$ & 1 & $\mathrm{NE}$ & 1 & $\mathrm{CT}+\mathrm{NE}$ & 1 \\
\hline $\mathrm{CT} * \mathrm{NE}$ & 1.82 & $\mathrm{CT} * \mathrm{NE}$ & 30 & $\mathrm{CT} * \mathrm{NE}$ & 31 \\
\hline All other models & $>100$ & All other models & $>100$ & All other models & $>100$ \\
\hline
\end{tabular}

Note. All Bayes Factors are relative to the best fitting model (i.e. in this case, the model of which the Bayes Factor is 1). All other models include all combinations from an empty to a full model not reported in the table. $\mathrm{CT}=$ Configuration Type; $\mathrm{NE}=$ Number of Elements

rotated stimulus configurations. Alternatively, if the single elements comprising each configuration were driving the configuration effect observed in Experiment 1, we predicted that the Number of Elements (full configuration vs. single element experiment) would not yield differential effects (interactions) with the Configuration Type.

The pattern of results that was observed in Experiment 2 could not be simply interpreted in either direction. That is, the stimuli that were generated for the scrambled condition yielded longer suppression times for the traditional pacmen only. In the case of the cross stimulus, the scrambled configuration yielded shorter suppression times compared to the rotated configuration. For the cross, this pattern also was observed in the single element session. However, for the other two conditions (traditional and curved pacmen), the single element session was less readily comparable with the full configuration session. For the curved pacmen, the BF analysis indicated strong evidence for a main effect of Number of Elements only, indicating no differential pattern of results across both sessions (yet also no effect of Configuration Type). For the traditional pacmen, the BF analysis indicated a potential interaction between Configuration Type and Number of Elements, although the results obtained in both sessions were qualitatively similar. When this interaction was further resolved, an effect of configuration was obtained only in the full configuration session. Furthermore, when the data were analyzed for the subset of stimuli used in Experiment 1 only, an interaction between configuration and Stimulus Type was observed. This interaction was primarily driven by the absence of a configuration effect for the curved pacmen. Thus, compared to Experiment 1, this subset of the data now indicated that aligned edges in the stimulus were primarily driving the configuration effect.

What is the most parsimonious interpretation of these results given the hypotheses laid out at the start of Experiment 2 and the results that were observed? We have clearly replicated one aspect of our findings from Experiment 1, namely that the ability for the stimuli to evoke a Kanizsa surface percept plays no role in breakthrough times. The fact that there was no consistent influence of scrambling for the three Stimulus Types also makes it hard to justify an effect based on the global configuration. On the one hand, there were some differences between the single element and full-configuration conditions, so the results in the full configurations cannot be fully predicted by the effects for the single elements. On the other hand, there was no interaction between Configuration Type and Number of Elements for the curved pacmen and the cross conditions, while qualitatively similar results were obtained for the full and single-element stimuli in the traditional pacmen condition. All in all, an account based on the features present in the individual elements and across the configuration as a whole seems to offer the most likely explanation for the effects of configuration observed here and in Experiment 1. Reflecting retrospectively on our results, it is worth pointing out that the visual system is most sensitive to cardinal orientations (horizontal and vertical) and less so to oblique ones (Appelle, 1972; Campbell \& Kulikowski, 1966; Li et al., 2003; Yacoub et al., 2008). From this perspective, the configuration effects observed in both experiments could be explained potentially by the differences between the orientations most prominently present in the stimuli, at the spatial scales of the individual elements and of the configuration as a whole. Indeed, for all Stimulus Types, the standard stimuli show strong peaks at the cardinal orientations in the Fourier orientation spectrum, compared to the rotated and scrambled stimuli (Fig. 4). This difference at the cardinal orientations is most evident in the cross and traditional pacmen conditions, which can explain the consistent differences observed between the standard and rotated configurations. The configurations for the curved pacmen, on the other hand, show considerable overlap, which could explain why the results obtained for the curved pacmen condition are less consistent between experiments.

The stimuli that were generated for the scrambled condition include orientations in-between cardinal and oblique orientations, which is most pronounced in the orientation spectrum of the cross stimulus. In this respect, it makes sense that the scrambled cross configuration broke suppression faster compared to the rotated one (and similarly so for the single element condition). Furthermore, in the case of the single element condition, the orientation spectra of both the traditional and curved pacmen show strong overlap for all different 

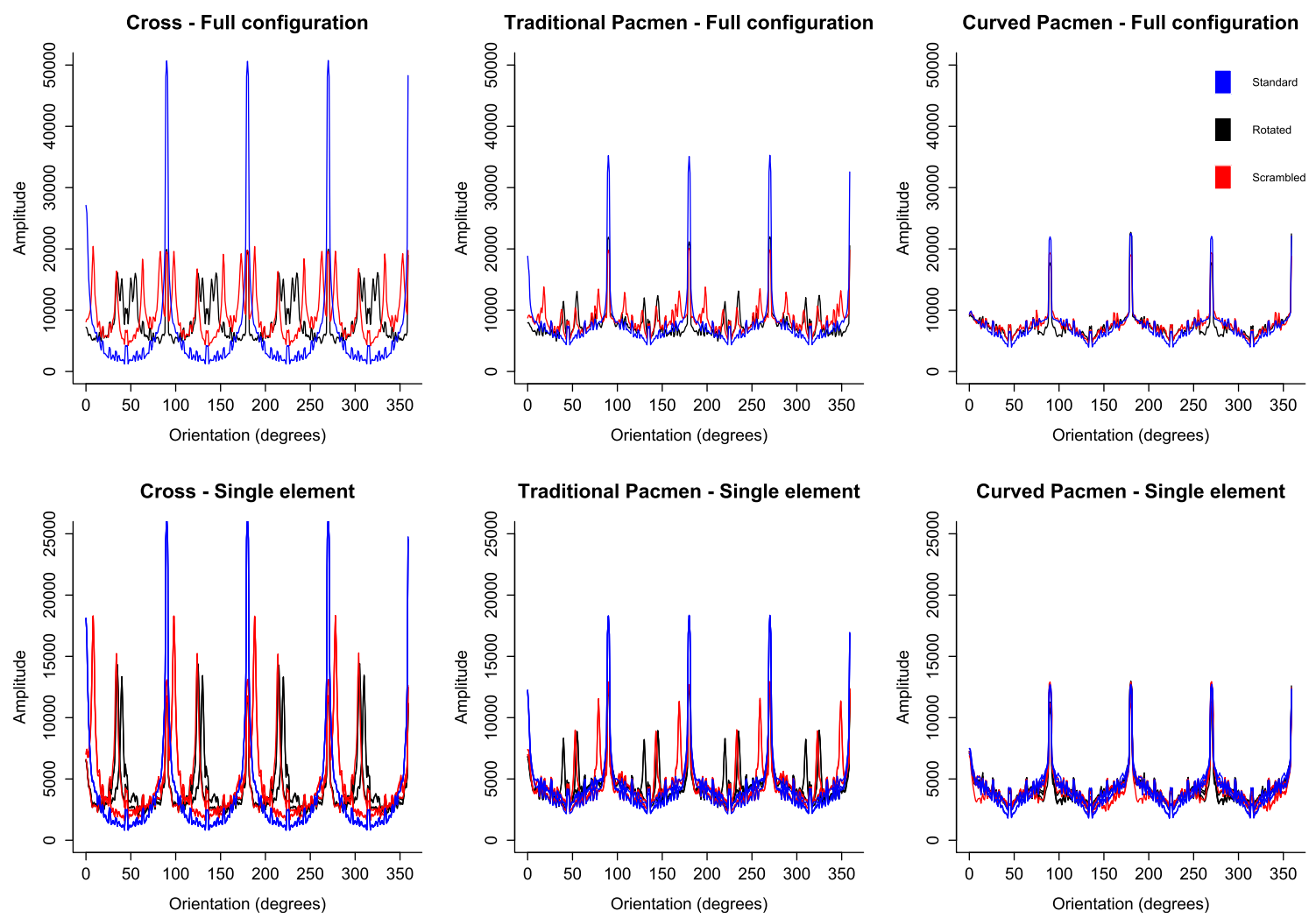

Fig. 4 Fourier orientation spectrum for all stimuli. A fast Fourier transform was applied to all stimuli and the orientation spectrum was obtained by averaging the amplitude spectrum across all spatial frequencies for each orientation, separately

configurations, which might explain the absence of a configuration effect for these stimuli in the single element condition. Moreover, one has to take into account that the visual inputs vary considerably between the full configuration and single elements, making it more difficult to obtain significant effects from potentially subtle differences between stimuli for the latter condition (unless they are strongly represented as in the cross configurations).

\section{General discussion}

In this study, we set out to test whether the advantage of a traditional pacmen stimulus in breaking CFS (compared to a rotated control configuration) was due to the ability of that configuration to induce the perception of a surface or the existence of collinear edges in the image. Experiment 1 was a conceptual replication and extension of Wang et al. (2012). In addition to a traditional pacmen stimulus, two types of control stimuli were included. A cross configuration was used to assess whether the suppression time benefit of traditional pacmen configurations could be caused by the alignment of the edges rather than the ability for the stimulus to elicit a surface percept. Additionally, we included a configuration that consisted of curved pacmen. This stimulus elicited a surface percept but, critically, none of the contours of the inducers were collinear with each other. The use of these two types of control stimuli enabled us to test whether the suppression time difference observed in Wang et al. (2012) could be explained by a genuine surface-based figure-ground assignment process (evident in the curved pacmen stimulus) or rather by the local alignment of edges in the inducers (evident in the cross stimulus). The results of Experiment 1 showed a clear replication of the suppression time difference between standard and rotated traditional pacmen stimuli observed in Wang et al. (2012), but this effect did not distinguish between the stimulus types employed. That is, in all conditions a difference between a standard and rotated global configuration was observed. This observation indicates that the suppression time difference in the traditional pacmen condition was not specific to the potential for the stimulus to induce a surface percept.

As a follow-up, we conducted a second experiment in which we explored whether the global configuration was driving the observed differences between conditions in Experiment 1 or, alternatively, that the differences were being driven by the differences between the single elements belonging to these configurations. To this end, we used the same stimuli as in Experiment 1 with the addition of a set of scrambled stimuli (by manipulating the distance and orientation of the individual elements relative to the fixation cross, as well as their individual orientation) and tested participants in two sessions. In one of them, the full configurations were used as 
stimuli. In the other, on each trial a single element of one of the configurations was presented. If the configuration effect observed in Experiment 1 was due to the global stimulus configuration, we predicted that the scrambled stimulus configuration would elicit slower suppression times compared to the rotated configuration. Alternatively, if the elements of the configurations were driving the differences, we predicted to observe a similar pattern of results in both full and singleelement experimental sessions.

The results revealed no consistent longer suppression durations for the scrambled configurations compared to the standard and rotated ones. Furthermore, although the data were qualitatively similar between the full configuration and single element sessions (except for the curved pacmen), no conclusive evidence was obtained that the results of the single element session were the same as in the full configuration session. Based on these results, we argued that a possible account for the data could be the differences in low-level stimulus features such as orientation. That is, cardinal orientationsto which the visual system is most sensitive-were more strongly represented in the stimuli that broke suppression fastest and these differences were most pronounced in the full configuration experiment because of the presentation of the simultaneous presentation of four rather than one element.

Our study was motivated by the observation that there was a discrepancy between the findings of Sobel and Blake (2003) and Harris et al. (2011) on the one hand and Wang et al. (2012) on the other. Although we replicated the findings reported in the latter study, the overall pattern of our results clearly supports the conclusions advanced in the former studies. That is, our results fail to provide evidence that, during CFS, traditional pacmen stimuli can induce figure-ground processes that might lead to a differential effect for stimuli able to induce a surface percept. This observation is consistent with a broader set of recent studies focusing on the extent to which mid- and high-level stimuli are represented during CFS (Faivre \& Koch, 2014; Gayet, Van Der Stigchel, \& Paffen, 2014; Hedger, Adams, \& Garner, 2015; Hesselmann \& Knops, 2014; Hesselmann \& Moors, 2015; Heyman \& Moors, 2014; Moors, Huygelier, Wagemans, de-Wit, \& van Ee, 2015). That is, there is converging evidence that suppressed stimuli are processed to a limited extent during CFS and that any process that requires complex integration of several features of the suppressed stimulus is unlikely to take place. Given that the construction of a Kanizsa surface percept not only requires grouping, but also figure-ground assignment, it is logical to predict that it should not manifest under CFS. This is furthermore reinforced by the studies reporting on neural activity associated with stimuli suppressed during CFS (Fogelson, Kohler, Miller, Granger, \& Tse, 2014; Hesselmann \& Malach, 2011; Ludwig, Kathmann, Sterzer, \& Hesselmann, 2014; Sterzer, Stein,
Ludwig, Rothkirch, \& Hesselmann, 2014; YuvalGreenberg \& Heeger, 2013). Invariably, these studies show that activity related to the suppressed stimulus is limited to early visual areas such as V1 and V2. Parallel work on the Kanizsa stimulus, from functional neuroimaging studies (Seghier \& Vuilleumier, 2006; Stanley \& Rubin, 2003), neuropsychological research (de-Wit et al., 2009), and comparative studies with monkeys (Huxlin, Saunders, Marchionini, Pham, \& Merigan, 2000) suggests that higher areas in the ventral stream (and the lateral occipital complex in particular) are critically involved in the construction of the surface percept for traditional pacmen stimuli. Thus, combining this knowledge of the extent to which CFS should block information transfer to higher areas, and the role of higher ventral areas in the construction of the Kanizsa surface percept the results reported in this study are not surprising. Furthermore, it highlights the importance of using a range of control conditions to be sure that any difference observed between experimental conditions is actually the result of the perceptual difference elicited by the stimuli used in those conditions, especially for perceptually compelling phenomena like the Kanizsa surface.

Given that the relationship between perceptual organization and awareness necessarily involves the choice of a suitable paradigm to render a stimulus invisible, it remains plausible that Kanizsa surfaces can be constructed in the absence of visual awareness depending on the nature of suppression of the paradigm that was employed (Breitmeyer, 2015; Breitmeyer, Koç, Oğmen, \& Ziegler, 2008; Hesselmann \& Moors, 2015). Indeed, using visual masking, a paradigm for which it has been argued that the initial feedforward transfer of input to higher areas, there is evidence that masked Kanizsa like stimuli can influence performance on a subsequent shape discrimination task (Poscoliero, Marzi, \& Girelli, 2013). Similarly, a recent neuroimaging study relying on an inattentional blindness paradigm reported evidence for similar processing of Kanizsa figures for groups of participants that either could or could not distinguish a Kanizsa figure in a forced-choice task after having performed an attentionally demanding task (and, hence, had been inattentionally blind to the Kanizsa figure). Moreover, neuropsychological studies relying on patients suffering from visuospatial neglect, a condition mainly caused by stroke in the parietal region, also provided evidence for processing of illusory shapes and contours (Conci et al., 2009; Driver \& Mattingley, 1998; Mattingley, Davis, \& Driver, 1997).

Thus, the main message of this study is not necessarily that one needs to be aware of a visual stimulus for some form of perceptual grouping and figure-ground assignment to take place, but that one needs to consider the specific mechanisms by which a particular paradigm renders stimuli invisible. Depending on the level at which suppression takes place, one might reasonably hypothesize that a process can happen 
in the absence of visual awareness. For CFS or other binocular suppression techniques, however, it seems highly likely that the processing of a suppressed stimulus is rather limited.

Acknowledgments The authors thank San Verhavert for help in the data collection. PM is supported by the Fund for Scientific Research Flanders (FWO) through a doctoral fellowship. JW is supported by the Methusalem program of the Flemish Government (METH/08/02 and METH/14/02). RVE is supported by FWO and the EU Horizon 2020 program (HealthPac). LDW is supported by FWO through a postdoctoral fellowship.

\section{References}

Alais, D., \& Blake, R. (2015). Binocular rivalry and perceptual ambiguity. In J. Wagemans (Ed.), Oxford Handbook of Perceptual Organization. Oxford: Oxford University Press.

Appelle, S. (1972). Perception and discrimination as a function of stimulus orientation: The "oblique effect" in man and animals. Psychological Bulletin, 78(4), 266-278.

Breitmeyer, B. G. (2015). Psychophysical "blinding" methods reveal a functional hierarchy of unconscious visual processing. Consciousness and Cognition. doi:10.1016/j.concog.2015.01.012

Breitmeyer, B. G., Koç, A., Oğmen, H., \& Ziegler, R. (2008). Functional hierarchies of nonconscious visual processing. Vision Research, 48(14), 1509-1513. doi:10.1016/j.visres.2008.03.015

Campbell, F. W., \& Kulikowski, J. J. (1966). Orientational selectivity of the human visual system. The Journal of Physiology, 187(2), 437445. doi:10.1113/jphysiol.1966.sp008101

Conci, M., Böbel, E., Matthias, E., Keller, I., Müller, H. J., \& Finke, K. (2009). Preattentive surface and contour grouping in Kanizsa figures: Evidence from parietal extinction. Neuropsychologia, 47(3), 726-732. doi:10.1016/j.neuropsychologia.2008.11.029

Cox, M. A., \& Maier, A. (2015). Serial versus parallel processing in midlevel vision: Filling-in the details of spatial interpolation. Neuroscience of Consciousness, 2015(1), 1-7.

de-Wit, L. H., Kentridge, R. W., \& Milner, A. D. (2009). Shape processing area LO and illusory contours. Perception, 38(8), 1260-1263.

Driver, J., \& Mattingley, J. B. (1998). Parietal neglect and visual awareness. Nature Neuroscience, 1(1), 17-22. doi:10.1038/217

Faivre, N., \& Koch, C. (2014). Inferring the direction of implied motion depends on visual awareness. Journal of Vision, 14(4), 4.

Fang, F., \& He, S. (2005). Cortical responses to invisible objects in the human dorsal and ventral pathways. Nature Neuroscience, $8(10)$, 1380-1385. doi:10.1038/nn1537

Fogelson, S. V., Kohler, P. J., Miller, K. J., Granger, R., \& Tse, P. U. (2014). Unconscious neural processing differs with method used to render stimuli invisible. Frontiers in Psychology, 5, 601. doi:10. 3389/fpsyg.2014.00601

Gayet, S., Van Der Stigchel, S., \& Paffen, C. (2014). Breaking continuous flash suppression: Competing for consciousness on the pre-semantic battlefield. Frontiers in Psychology, 5(460). doi:10.3389/fpsyg. 2014.00460

Gillebert, C. R., \& Humphreys, G. W. (2015). Mutual interplay between perceptual organization and attention: A neuropsychological perspective. In J. Wagemans (Ed.), Oxford Handbook of Perceptual Organization. Oxford: Oxford University Press.

Harris, J. J., Schwarzkopf, D. S., Song, C., Bahrami, B., \& Rees, G. (2011). Contextual illusions reveal the limit of unconscious visual processing. Psychological Science, 22(3), 399-405. doi:10.1177/ 0956797611399293
Hedger, N., Adams, W. J., \& Garner, M. (2015). Autonomic arousal and attentional orienting to visual threat are predicted by awareness. Journal of Experimental Psychology: Human Perception and Performance, 41(3), 798-806.

Hesselmann, G., \& Knops, A. (2014). No conclusive evidence for numerical priming under interocular suppression. Psychological Science. doi: $10.1177 / 0956797614548876$

Hesselmann, G., \& Malach, R. (2011). The link between fMRI-BOLD activation and perceptual awareness is "stream-invariant" in the human visual system. Cerebral Cortex, 21(12), 2829-2837. doi:10. 1093/cercor/bhr085

Hesselmann, G., \& Moors, P. (2015). Definitely maybe: Can unconscious processes perform the same functions as conscious processes? Frontiers in Psychology, 6(584). doi:10.3389/fpsyg.2015.00584

Heyman, T., \& Moors, P. (2014). Frequent words do not break continuous flash suppression differently from infrequent or nonexistent words: Implications for semantic processing of words in the absence of awareness. PLoS ONE, 9(8), e104719. doi:10.1371/journal.pone. 0104719

Hong, S. W., \& Blake, R. (2009). Interocular suppression differentially affects achromatic and chromatic mechanisms. Attention, Perception, \& Psychophysics, 71(2), 403-411. doi:10.3758/APP. 71.2.403

Huxlin, K. R., Saunders, R. C., Marchionini, D., Pham, H. A., \& Merigan, W. H. (2000). Perceptual deficits after lesions of inferotemporal cortex in macaques. Cerebral Cortex, 10(7), 671683.

Jeffreys, H. (1961). Theory of Probability. Oxford: Oxford University Press.

Kimchi, R. (2009). Perceptual organization and visual attention. Progress in Brain Research, 176, 15-33. doi:10.1016/S0079-6123(09) 17602-1

Kogo, N., Strecha, C., Van Gool, L., \& Wagemans, J. (2010). Surface construction by a 2-D differentiation-integration process: A neurocomputational model for perceived border ownership, depth, and lightness in Kanizsa figures. Psychological Review, 117(2), 406-439. doi:10.1037/a0019076

Kogo, N., \& Wagemans, J. (2013). The "side" matters: How configurality is reflected in completion. Cognitive Neuroscience, 4(1), 31-45. doi: 10.1080/17588928.2012.727387

Lee, T. S., \& Nguyen, M. (2001). Dynamics of subjective contour formation in the early visual cortex. Proceedings of the National Academy of Sciences of the United States of America, 98(4), 1907-1911. doi: 10.1073/pnas.031579998

Li, B., Peterson, M. R., \& Freeman, R. D. (2003). Oblique effect: a neural basis in the visual cortex. Journal of Neurophysiology, 90(1), 204217. doi:10.1152/jn.00954.2002

Ludwig, K., Kathmann, N., Sterzer, P., \& Hesselmann, G. (2014). Investigating category- and shape-selective neural processing in ventral and dorsal visual stream under interocular suppression. Human Brain Mapping. doi:10.1002/hbm.22618

Machilsen, B., \& Wagemans, J. (2011). Integration of contour and surface information in shape detection. Vision Research, 51(1), 179-186. doi:10.1016/j.visres.2010.11.005

Maehara, G., Huang, P.-C., \& Hess, R. F. (2009). Importance of phase alignment for interocular suppression. Vision Research, 49(14), 1838-1847. doi:10.1016/j.visres.2009.04.020

Mattingley, J. B., Davis, G., \& Driver, J. (1997). Preattentive filling-in of visual surfaces in parietal extinction. Science, 275(5300), 671-674.

Mitroff, S. R., \& Scholl, B. J. (2005). Forming and updating object representations without awareness: Evidence from motion-induced blindness. Vision Research, 45(8), 961-967. doi:10.1016/j.visres. 2004.09.044

Montoro, P. R., Luna, D., \& Ortells, J. J. (2014). Subliminal Gestalt grouping: Evidence of perceptual grouping by proximity and 
similarity in absence of conscious perception. Consciousness and Cognition, 25, 1-8. doi:10.1016/j.concog.2014.01.004

Moore, C. M., \& Egeth, H. (1997). Perception without attention: Evidence of grouping under conditions of inattention. Journal of Experimental Psychology. Human Perception and Performance, 23(2), 339-352.

Moors, P., Huygelier, H., Wagemans, J., de-Wit, L., \& van Ee, R. (2015). Suppressed visual looming stimuli are not integrated with auditory looming signals: Evidence from continuous flash suppression. $I$ Perception, 6(1), 48-62. doi:10.1068/i0678

Moors, P., Wagemans, J., \& de-Wit, L. (2014). Moving stimuli are less effectively masked using traditional continuous flash suppression (CFS) compared to a moving mondrian mask (MMM): A test case for feature-selective suppression and retinotopic adaptation. PLoS ONE, 9(5), e98298. doi:10.1371/journal.pone.0098298

Morey, R. D. (2008). Confidence intervals from normalized data: A correction to Cousineau (2005). Tutorials in Quantitative Methods for Psychology, 4, 61-64.

Morey, R. D., \& Rouder, J. N. (2015). BayesFactor: 0.9.11-1 CRAN [Zenodo]. Retrieved from http://doi.org/10.5281/zenodo.16238

Norman, L. J., Heywood, C. A., \& Kentridge, R. W. (2013). Object-based attention without awareness. Psychological Science, 24(6), 836843. doi:10.1177/0956797612461449

Palmer, S. E. (1999). Vision science: Photons to phenomenology (1st ed.). Cambridge: MIT Press.

Peirce, J. W. (2007). PsychoPy-Psychophysics software in Python. Journal of Neuroscience Methods, 162(1-2), 8-13. doi:10.1016/j. jneumeth.2006.11.017

Peirce, J. W. (2009). Generating stimuli for neuroscience using PsychoPy. Frontiers in Neuroinformatics, 2(10). doi:10.3389/neuro.11.010. 2008

Poort, J., Raudies, F., Wannig, A., Lamme, V. A. F., Neumann, H., \& Roelfsema, P. R. (2012). The role of attention in figure-ground segregation in areas V1 and V4 of the visual cortex. Neuron, 75(1), 143-156. doi:10.1016/j.neuron.2012.04.032

Poscoliero, T., Marzi, C. A., \& Girelli, M. (2013). Unconscious priming by illusory figures: The role of the salient region. Journal of Vision, 13(5), 27. doi:10.1167/13.5.27

Rouder, J. N., \& Morey, R. D. (2012). Default Bayes factors for model selection in regression. Multivariate Behavioral Research, 47(6), 877-903. doi:10.1080/00273171.2012.734737

Rouder, J. N., Morey, R. D., Speckman, P. L., \& Province, J. M. (2012). Default Bayes factors for ANOVA designs. Journal of Mathematical Psychology, 56(5), 356-374. doi:10.1016/j.jmp. 2012.08.001

Rouder, J. N., Speckman, P. L., Sun, D., Morey, R. D., \& Iverson, G. (2009). Bayesian $t$ tests for accepting and rejecting the null hypothesis. Psychonomic Bulletin \& Review, 16(2), 225-237. doi:10.3758/ PBR.16.2.225

Schwarzkopf, S. D., \& Rees, G. (2015). Perceptual organization and consciousness. In J. Wagemans (Ed.), Oxford Handbook of Perceptual Organization. Oxford: Oxford University Press.

Seghier, M. L., \& Vuilleumier, P. (2006). Functional neuroimaging findings on the human perception of illusory contours. Neuroscience and Biobehavioral Reviews, 30(5), 595-612. doi:10.1016/j. neubiorev.2005.11.002
Sobel, K. V., \& Blake, R. (2003). Subjective contours and binocular rivalry suppression. Vision Research, 43(14), 1533-1540.

Stanley, D. A., \& Rubin, N. (2003). fMRI activation in response to illusory contours and salient regions in the human lateral occipital complex. Neuron, 37(2), 323-331. doi:10.1016/S08966273(02)01148-0

Stein, T., \& Sterzer, P. (2014). Unconscious processing under interocular suppression: Getting the right measure. Frontiers in Psychology, 5, 387. doi:10.3389/fpsyg. 2014.00387

Sterzer, P., Stein, T., Ludwig, K., Rothkirch, M., \& Hesselmann, G. (2014). Neural processing of visual information under interocular suppression: A critical review. Frontiers in Psychology, 5, 453. doi: 10.3389/fpsyg.2014.00453

Tsuchiya, N., \& Koch, C. (2005). Continuous flash suppression reduces negative afterimages. Nature Neuroscience, 8(8), 1096-1101. doi: $10.1038 / \mathrm{nn} 1500$

Vancleef, K., Acke, E., Torfs, K., Demeyere, N., Lafosse, C., Humphreys, G., ... de-Wit, L. (2015). Reliability and validity of the Leuven Perceptual Organization Screening Test (L-POST). Journal of Neuropsychology, 9(2), 271-298. doi:10.1111/jnp.12050

Vancleef, K., Wagemans, J., \& Humphreys, G. W. (2013). Impaired texture segregation but spared contour integration following damage to right posterior parietal cortex. Experimental Brain Research, 230(1), 41-57. doi:10.1007/s00221-013-3629-7

Von der Heydt, R., Peterhans, E., \& Baumgartner, G. (1984). Illusory contours and cortical neuron responses. Science, 224(4654), 1260 1262. doi:10.1126/science.6539501

Wagemans, J., Elder, J. H., Kubovy, M., Palmer, S. E., Peterson, M. A., Singh, M., \& von der Heydt, R. (2012). A century of Gestalt psychology in visual perception: I. Perceptual grouping and figureground organization. Psychological Bulletin, 138(6), 1172-1217. doi: $10.1037 / \mathrm{a} 0029333$

Wang, L., Weng, X., \& He, S. (2012). Perceptual grouping without awareness: Superiority of Kanizsa triangle in breaking interocular suppression. PLoS ONE, 7(6), e40106. doi:10.1371/journal.pone. 0040106

Wokke, M. E., Vandenbroucke, A., Scholte, H. S., \& Lamme, V. A. F. (2013). Confuse your illusion: Feedback to early visual cortex contributes to perceptual completion. Psychological Science, 24(1), 6371. doi: $10.1177 / 0956797612449175$

Yacoub, E., Harel, N., \& Ugurbil, K. (2008). High-field fMRI unveils orientation columns in humans. Proceedings of the National Academy of Sciences of the United States of America, 105(30), 10607-10612. doi:10.1073/pnas.0804110105

Yang, E., \& Blake, R. (2012). Deconstructing continuous flash suppression. Journal of Vision, 12(3), 1-14. doi:10.1167/12.3.8

Yang, E., Blake, R., \& McDonald, J. E. (2010). A new interocular suppression technique for measuring sensory eye dominance. Investigative Ophthalmology \& Visual Science, 51(1), 588-593. doi:10.1167/iovs.08-3076

Yuval-Greenberg, S., \& Heeger, D. J. (2013). Continuous flash suppression modulates cortical activity in early visual cortex. The Journal of Neuroscience, 33(23), 9635-9643. doi:10.1523/JNEUROSCI. 4612-12.2013 